
approches théoriques et méthodologiques

\title{
Le code-barres 2D, du support marketing au medium
}

Two-Dimensional Barcodes: From Marketing Support to Medium

\section{Véronique Madelon}

\section{(2) OpenEdition}

\section{Journals}

Édition électronique

URL : http://journals.openedition.org/edc/3892

DOI : 10.4000/edc.3892

ISSN : 2101-0366

Éditeur

Université Lille-3

\section{Édition imprimée}

Date de publication : 1 décembre 2012

Pagination : 217-233

ISBN : 978-2-917562-08-6

ISSN : $1270-6841$

\section{Référence électronique}

Véronique Madelon, « Le code-barres 2D, du support marketing au medium », Études de communication [En ligne], 39 | 2012, mis en ligne le 01 décembre 2014, consulté le 02 mai 2019. URL : http:// journals.openedition.org/edc/3892 ; DOI : 10.4000/edc.3892

Ce document a été généré automatiquement le 2 mai 2019.

(c) Tous droits réservés 


\title{
Le code-barres $2 \mathrm{D}$, du support marketing au medium
}

Two-Dimensional Barcodes: From Marketing Support to Medium

\author{
Véronique Madelon
}

1 Selon une étude de l'AFMM ${ }^{1}$ menée en 2010, plus de la moitié des Français (57,8\%) connait les codes-barres 2D, c'est-à-dire sait de quoi il s'agit, mais beaucoup moins les utilise. Au Japon, ces signes font désormais partie du quotidien : d'après une étude publiée en octobre 2006 dans le livre blanc du Mobile Content Forum ${ }^{2}$ 83,6 \% de la population en auraient déjà «flashé » un. Inventés dans les années 90 par l'entreprise Denso-Wave, les codes-barres 2D sont des suites de carrés noirs et blancs représentant des 0 et des 1 , codées en langage binaire. Ils ont d'abord servi à suivre les pièces automobiles dans les usines Toyota. À partir des années 2000, et avec le développement des téléphones portables multimédias, leur fonction évolue. Les codes-barres 2D se diffusent plus largement et se tournent vers le public. Il en existe plusieurs types, les uns nécessitent une application payante pour les générer comme pour les décoder (les flashcodes sont la propriété d'Orange) et les autres sont totalement gratuits (QR-codes, Datamatrix, Aztec...). En fonction du type de codage utilisé, un même message produit des codesbarres 2D d'aspect différent (figure 1).

Figure 1 : exemples de codes-barre 2D

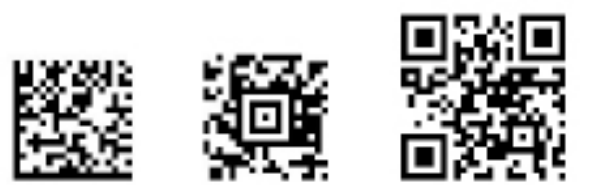

Datamatrix, Aztec et QR-code contenant le texte « du signe au medium »

2 Ces signes nécessitent l'utilisation d'un téléphone portable pouvant les photographier ( flasher », "scanner ») puis les décoder grâce à une application, déjà installée ou non, 
sur le portable. Ils sont ensuite envoyés par SMS ou Internet et servent à se connecter à un site Internet Mobile, envoyer un SMS, un MMS ou un email, générer un appel téléphonique, etc... Ils apparaissent alors essentiellement dans les publicités mais sont aussi le support d'une communication interpersonnelle : pouvant être produits par tout un chacun ${ }^{3}$, des utilisateurs choisissent de faire passer des messages par leur biais ${ }^{4}$. En France, les codes-barres 2D sont de plus en plus visibles : ils s'insèrent dans des supports traditionnels plus anciens comme les billets de train, les relevés de compte, les affichages informationnels des abribus... Ils prennent même parfois la place des légendes accompagnant les œuvres d'art dans des expositions. La multiplicité des fonctions comme des usages de cet objet nous pousse à nous interroger sur l'évolution de son statut et des valeurs qui lui sont associées. Adoptant dans un premier temps une approche sémiotique, nous nous interrogeons sur sa qualité de medium. Partant des conséquences de cette caractéristique sur les enjeux de réception, nous nous questionnerons ensuite sur les valeurs qui peuvent lui être attribuées, valeurs intimement liées aux pratiques entourant cette nouvelle technologie.

\section{Signe, support, medium?}

3 Avant toute chose, posons nous la question du statut de cet objet : relève t-il du signe, du support ou du medium ? Le code-barres 2D est un signe en ce qu'il est là pour représenter, rendre présent autre chose, il devient support si l'on considère le regard que l'on porte sur lui, les valeurs qu'on décide de lui attribuer (Quinton, 2008). Mais il faudra parler de medium à partir du moment où le code-barres 2D crée du lien entre la production et la réception (Mitropoulou, 2008). Le medium est « un instrument de communication qui transporte dans l'espace et dans le temps un message incarné dans un langage donné » (Cloutier, 1973, p.172) et surtout qui prédéfinit pour partie les conditions et le programme de réception. En somme, c'est le regard de celui qui le perçoit qui va conditionner son statut ${ }^{5}$. Dérivé du code-barres classique, le code-barres $2 \mathrm{D}$ permet d'encoder environ 300 fois plus d'informations et ce, de manière plus compacte. Activer un code-barres 2D, c'est faire un lien entre un objet réel et un contenu virtuel mis à la disposition de l'utilisateur. Mais est-ce suffisant pour le considérer comme un medium ? Selon E. Mitropoulou (2008), on peut parler de l'irruption d'un nouveau ${ }^{6}$ medium lorsqu'il y a nouvelle pratique médiatique et celle-ci est engendrée par la présence de trois ruptures (par rapport aux media en place) conjointes: une rupture du processus technologique, une rupture du faire réceptif et une rupture de l'expérience médiatique. "Si les trois ruptures sont actives 'il y a' irruption d'un nouveau medium, sinon 'il y a' simulacre d'irruption donc simulacre d'un nouveau medium» (Mitropoulou, 2008). Autrement dit, «le medium est ce qui introduit une rupture sémio-communicationnelle, propre au contrat axiologique de l'échange, que cette rupture soit effective ou simulée » ( op.cit.). L'intérêt pour nous de cette définition, est qu'elle donne des indications sur la manière dont on peut appréhender un médium. Ainsi, un médium peut s'envisager selon trois axes (technologie, réception, expérience), et lorsque l'analyse met en évidence une rupture sur ces trois axes, l'objet éttudié peut être considéré comme un medium. Il s'agit pour nous de confronter le code-barres 2D à ces différents axes pour en souligner le caractère de medium, pour souligner aussi sa dimension novatrice par rapport à des médias plus traditionnels (raison pour laquelle nous avons conservé cette idée de rupture), et enfin pour mettre en évidence les valeurs qui s'en dégagent. Nous nous 
appuierons pour cela sur l'étude de deux utilisations de cette technologie, à un an d'intervalle et dans des situations de communication relativement proches (promotion d'un festival et d'un concert dans la même ville).

\section{Au cœur du marketing : le flashcode}

Nous considérerons dans un premier temps un flashcode intégré dans le programme papier du festival bisontin L'Herbe en Zik, édition 2010. Nous procéderons à une analyse socio-sémiotique de cet objet et du contexte dans lequel il s'intègre, nous concentrant sur la production d'une mise en condition d'une réception, nous appuyant essentiellement sur les travaux de Courtès (1991). Nous voulons en effet démontrer que le flashcode est un medium, tel que nous l'avons défini précédemment. Ce flashcode est inséré dans un encart publicitaire pour Orange, entreprise qui l'a créé dans le but de mettre à disposition des festivaliers une version numérique du programme du festival. Cet exemple rend compte d'une imbrication complexe de plusieurs objets aux enjeux différents: dans un texte informatif et promotionnel (un programme papier), est inséré un texte publicitaire (une publicité papier pour Orange), dans lequel on peut voir un flashcode. Celui-ci est hermétique, il nécessite l'utilisation d'un téléphone récent possédant une caméra et un accès Internet mobile et il doit respecter certaines conditions techniques d'impression pour être lisible. Il renvoie alors à un autre contenu informatif supporté par l'Internet mobile. Cet encart présente, visuellement, les flashcodes (le signe est surmonté de son appellation). Il donne également la marche à suivre pour télécharger l'application capable de les décoder («1. envoyez 'FLASHCODE' par SMS [...]. Flashcode s'installe tout seul!»). Notons que cette application a le même nom que l'objet qu'elle est censée décoder, ce qui peut induire quelques confusions. Par contre, cet encart ne donne pas d'indication quant à l'utilisation même des flashcodes.

\section{Première rupture : passage du papier au numérique}

5 Le programme d'un festival est diffusé sur divers media et supports, aux enjeux relativement différents. La version papier (flyers, programme, affiche et même dossier de presse) est diffusée avec pour double enjeu de toucher et de séduire un public le plus large possible mais aussi de l'informer, de lui donner des indications précises sur les caractéristiques de l'événement, son déroulement... Sa version en ligne répond à ce même objectif d'information, s'adressant en priorité à un public déjà intéressé par l'événement (qui a fait la démarche d'aller chercher de l'information). Le flashcode crée un lien direct entre le support papier et le site Internet. Il permettrait donc d'avoir accès partout à des informations actualisées. En effet, les informations mises en ligne sont facilement modifiables (au contrai re de celles présentées sur un support papier). Aussi, le flashcode peut renvoyer sur des media aussi variés que la vidéo, les blogs, les sites Internet... Il a donc la potentialité d'offrir à ses utilisateurs des contenus enrichis et pluricodiques. Cependant, l'intérêt du flashcode reste à nuancer d'une part parce que toutes les possibilités offertes par cet objet ne sont pas forcément exploitées et d'autre part parce que l'information apportée par le flashcode risque d'être redondante par rapport au programme papier si aucune modification n'intervient dans la programmation. 


\section{Deuxième rupture : modification des habitudes de réception}

6 Le flashcode est d'abord vendu par les entreprises qui les commercialisent mais aussi par de simples prescripteurs comme un outil très pratique, une «solution ». Il est censé limiter le nombre de clics sur l'Internet mobile et ne nécessite pas d'accès Internet particulier? ${ }^{7}$. Le flashcode peut également renvoyer à un blog et mettre en place une certaine interactivité entre leurs utilisateurs et le producteur/diffuseur. Aussi, le flashcode pourrait être comparé, dans son fonctionnement, à un hyperlien : il donne la possibilité à son utilisateur de l'utiliser ou non et d'accéder à un supplément d'information. La potentialité du discours est donc dépendante de l'activation par l'utilisateur de cet objet. On peut cependant noter que le flashcode ne peut être décodé qu'avec un certain type de téléphone sur lequel il faut installer au préalable un lecteur de flashcode, puis qu'il faut envoyer un SMS. Le flashcode ne livre donc pas son message d'emblée. Il nécessite une participation relativement importante de l'utilisateur. Comme le note Fabien Eychenne (2009), ce sont les signes de l'existence d'un contenu numérique mais ce contenu numérique n'est en général révélé qu'au moment de son activation. En somme, l'utilisateur des codes-barres 2D ne sait pas toujours ce qu'il télécharge en terme de contenu et de nature. Dans ce sens, le flashcode, par son nom d'abord mais aussi par son fonctionnement, a clairement une dimension ludique, il rappelle les indices de jeux de piste qu'il faut trouver et décoder pour avancer dans le jeu. Cet objet aurait donc un fort pouvoir impliquant sur son utilisateur, ce dernier devant s'équiper technologiquement, rechercher les flashcodes, les décoder, les activer et même enrichir leur message par ses propres contributions. Cette observation fait écho aux recherches de A.-M. Dujarier (2008) sur la mise au travail du consommateur, mais nous reviendrons sur ce point infra.

\section{Troisième rupture : Incidences sur l'appropriation des discours}

7 Le flashcode, dans ce cadre particulier, projette sur le message auquel il donne accès différents niveaux de valeur. Il s'agit d'abord de valeurs liées à sa technicité : l'utilisation du flashcode n'est pas simple mais elle permet de simplifier l'utilisation de l'Internet mobile. Un autre aspect technologique est également "axiologisé » positivement: le flashcode permet un accès rapide à un grand nombre d'informations diversifiées. La valeur de base, informer, est supplantée par des valeurs d'usage: simplement, rapidement et en quantité, valeurs fréquemment associées à tout contenu numérique. A ceci s'ajoute d'autres valeurs, plus spécifiques au flashcode (et au code-barres 2D en général) : ce que Floch (1989) nomme valeur utopique, la proximité, et valeur ludique, l'expérimentation. En effet, la relation avec l'utilisateur est particulièrement mise en avant : d'abord, l'accès au message délivré par les flashcodes est limité aux initiés et aux équipés. Ensuite, cette relation nécessite une implication plus ou moins forte de l'utilisateur: il doit rechercher ces flashcodes, accepter de ne pas savoir exactement à quoi ils renvoient, les activer... Cette relation se base finalement sur un contrat fiduciaire : l'utilisateur se fie à une promesse plus ou moins explicite d'amélioration, de hausse de son savoir et donc de changement d'état lorsqu'il utilise les flashcodes. Le flashcode, comme tout code-barres 2D, répond donc aux trois logiques d'actions définies par F. Jauréguiberry et S. Proulx (2011) et motivant l'usage des nouvelles technologies: apporter des informations rapidement et en quantité ("être efficace»), renforcer l'appartenance à un groupe (" être branché ») tout en se démarquant (« être autonome »). 


\section{Ni seulement un signe, ni seulement un support}

Si les codes-barres 2D utilisés dans les usines Toyota ne sont que des supports d'informations, si ceux aperçus dans les abribus parisiens par un Français ne possédant pas de portable ne sont considérés que comme des signes, il est évident que dans la situation étudiée précédemment, le flashcode peut être pensé comme un medium : il sert de lien entre deux supports, modifie les habitudes de réception et a une influence sur l'appropriation du message; en effet, il se démarque des autres media et notamment de celui qui lui sert de support en renvoyant sur une autre technologie, en poussant son utilisateur à agir et en lui faisant croire que son action lui permettra d'obtenir un savoir supérieur aux non-utilisateurs. Le flashcode, mais finalement tout code-barres 2D, a un statut de medium et en cela, il permet d'instaurer de nouvelles stratégies communicationnelles à la frontière entre communication de masse et communication interpersonnelle: visible de tous, ils ne sont déchiffrables, ils ne délivrent leur message qu'aux initiés et seulement si ces derniers le décident.

\section{Créer l'événement}

En moins d'un an, les codes-barres 2D se sont multipliés. À Besançon, par exemple, où nous avons effectué nos observations, on en retrouve dans les vitrines d'agence immobilière, sur les affichages publics (figure 2)...

Figure 2 : exemples de codes-barre 2D (Besançon, mai 2011)
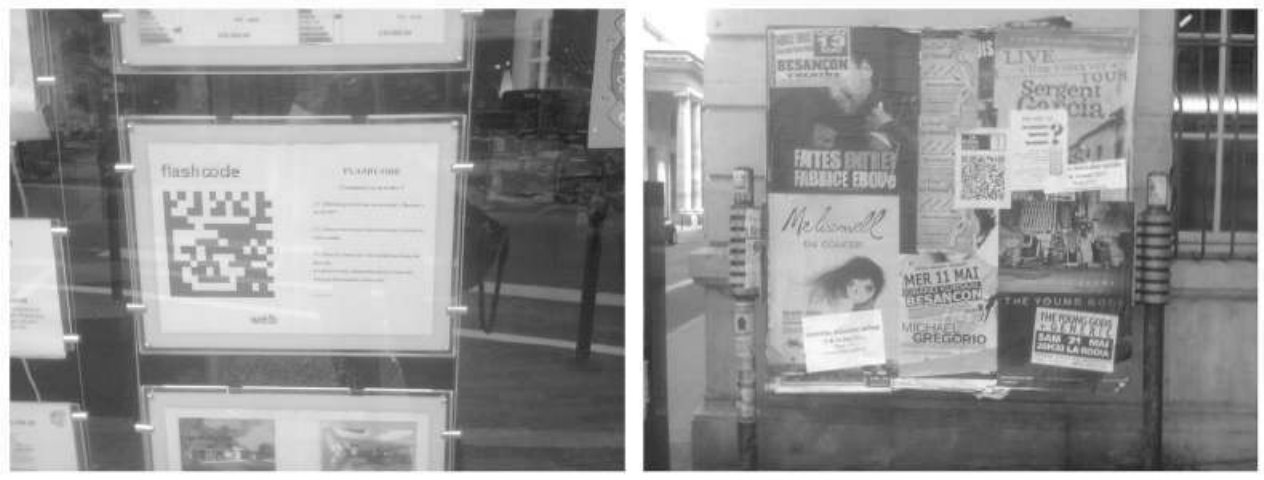

Nous avons été interpellée par la promotion de la création sonore et visuelle Machination ${ }^{8}$. Une campagne de communication de quatre semaines, basée sur l'édition de quatre QRCodes imprimés sur des affiches format A3, annonce cet événement. Nous envisageons à travers son étude, croisée avec les retours sur pratiques de Nicolas Chevailler ${ }^{9}$, graphiste concepteur de cette campagne, de déconstruire une utilisation relativement élaborée de cet objet. En effet, si l'exemple abordé supra peut être perçu comme un gadget, un " attrape-technophiles ", qu'en est-il d'un plan de communication entièrement pensée en fonction de cette technologie? Comment le concepteur de cette campagne va-t-il concilier l'aspect mystérieux et ludique de ce medium aux objectifs informationnels et prédicatifs de toute affiche de concert? Quelles valeurs lui sont associées, et que permettent-elles? 


\section{Une campagne de communication en quatre temps}

Figure 3 : première affiche de la campagne Machination

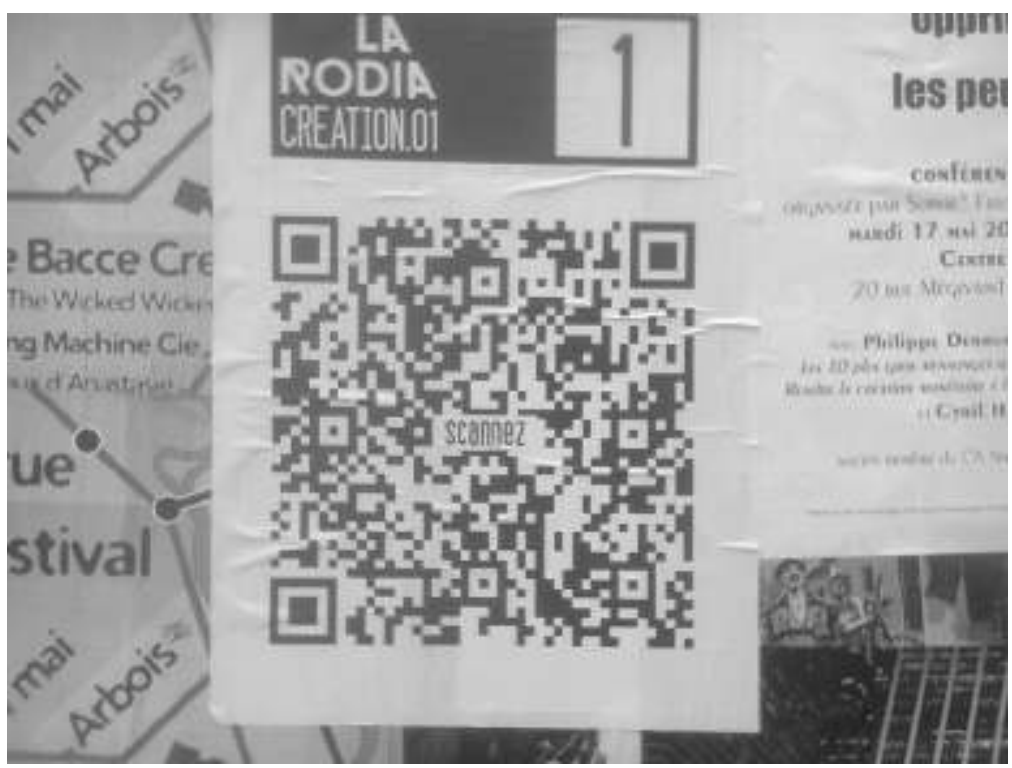

11 Le premier QR-code (figure 3) est diffusé trois semaines avant la présentation au public de la création. Alors que les bisontins ne sont encore pas très habitués à l'utilisation de ce type d'objet, il est accompagné de peu d'éléments: le nom de la salle apparait («LA RODIA ») ainsi que la mention "CREATION.01». L'association de ces deux termes laisse entendre qu'il peut s'agir d'un événement culturel, d'une création artistique, la première dans cette salle. Cette déduction est renforcée par le contexte dans lequel cette affiche apparaît (cf. figure 2), un panneau d'affichage dédié aux spectacles. Le chiffre « 1 » est explicitement mis en évidence. Dans un caractère très gros, encadré, il implique l'idée de suite. Enfin, le terme « scannez » est intégré au QR-code même. L'utilisation de l'impératif permet de s'adresser directement au lecteur, de lui donner une instruction. Cette affiche se distingue des affiches plus classiques, telles que celles étudiées par Fontanille (2004) par exemple, du fait de son opacité : elle annonce un événement culturel sans le nommer, sans en donner la date. Elle ne joue pas, a priori, sur une éventuelle identification ni même évaluation axiologique. Elle demande à son lecteur d'agir sans lui donner d'indication quant au résultat de son action et cette action (scanner le QR-code) donne accès de manière éphémère (le lien reste valide jusqu'à la date de la présentation publique) au teaser de la création. 
Figure 4 : deuxième affiche de la campagne

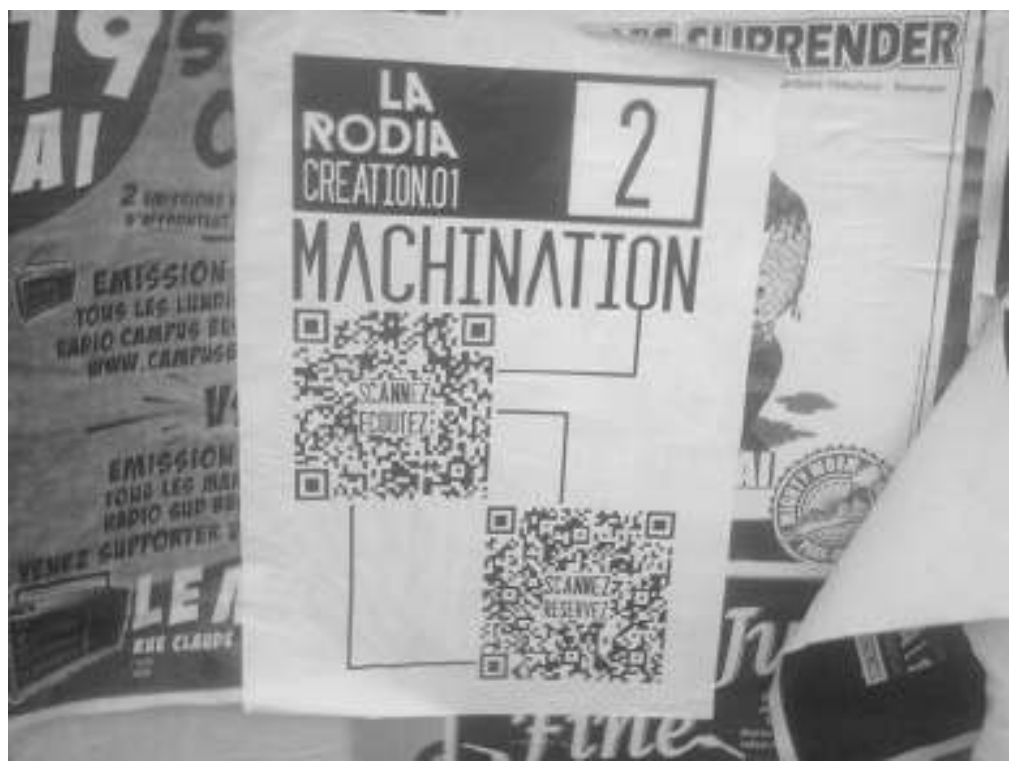

12 La deuxième affiche de la campagne Machination (figure 4) est collée en ville la semaine suivante. Elle comporte les mêmes informations que la précédente ainsi que le chiffre « 2 », elle indique cette fois le nom de l'événement en question «MACHINATION » et présente deux $\mathrm{QR}$-codes, l'un renvoyant sur un contenu à télécharger, en l'occurrence, un titre extrait de la création. Les termes «SCANNEZ ECOUTEZ» sont intégrés au medium. L'autre, accompagné des termes «SCANNEZ RESERVEZ », renvoie au site de la Rodia qui dispose d'une billetterie en ligne. Ces deux liens sont également éphémères et deviendront obsolètes après que l'évènement ait eu lieu.

Figure 5 : troisième affiche de la campagne Machination

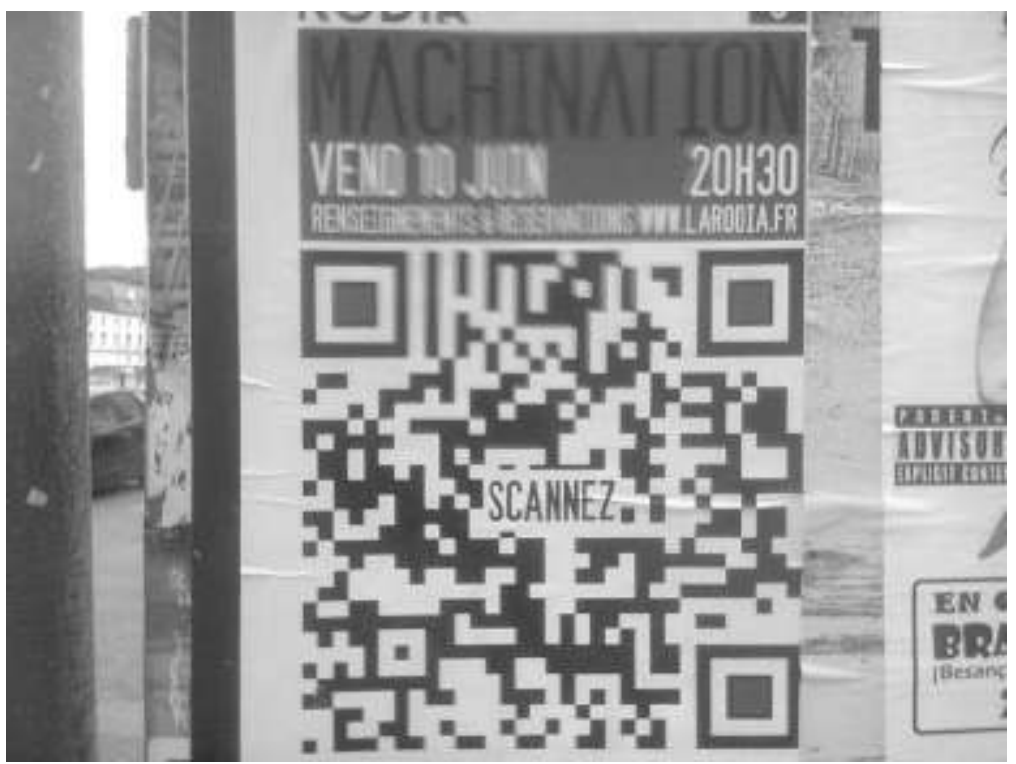

Le quatrième QR-code (figure 5) est diffusé une semaine avant l'événement. En plus des éléments apportés par l'affiche précédente, celle-ci indique la date et l'horaire de l'événement et donne l'adresse Internet de la salle de spectacle sur laquelle on peut 
trouver des renseignements concernant la création. Cette affiche se détache visuellement des deux précédentes car elle intègre des aplats rouges dans sa partie supérieure (consacrée aux informations linguistiques) mais également à l'intérieur du QR-code. Comme pour la première affiche, le QR-code occupe près de $60 \%$ de l'espace ${ }^{10}$. Il comporte également le terme "SCANNEZ». Mais cette fois, il renvoie au permalien du site Machination ${ }^{11}$. Ce site indique les noms des artistes concernés, présente en une phrase leur réalisation, donne rapidement quelques informations sur chacun de ses acteurs et comporte un lien hypertextuel vers les sites de chacun d'eux.

La quatrième et dernière affiche (figure 6) reprend le quatrième QR-code dans un format plus réduit (il occupe environ $15 \%$ de l'espace) et sans le terme "SCANNEZ ». Elle est également diffusée une semaine avant la représentation. La mise en page de cette affiche est relativement différente: la partie supérieure (nom de la création, lignes rouges et noms des artistes) reprend en partie la mise en page du site Internet. Le QR-code est relié par des lignes brisées (rappelant un circuit intégré) aux noms des musiciens ainsi qu'au nom de la salle et aux termes "CREATION.01 », inscrits cette fois dans une typographie réduite dans la partie inférieure de l'affiche. Enfin, les deux dates et lieux de représentation sont indiqués juste en dessous. Cette affiche est accompagnée d'un bandeau au format A4 répétant certaines informations (nom de l'événement, musiciens, un des deux lieux et l'une des deux dates) dans des caractères plus lisibles et précisant que cette création est le fruit de plusieurs partenariats. Cette dernière affiche, si elle fait la synthèse des précédentes, apportant toutes les informations nécessaires à la compréhension directe du message (du type : «Venez au concert »), minimise le rôle du QR-code, qui, sans instruction, est réduit pour les non-initiés à une représentation abstraite, une illustration de la thématique de la création.

Figure 6 : Quatrième affiche de la campagne Machination
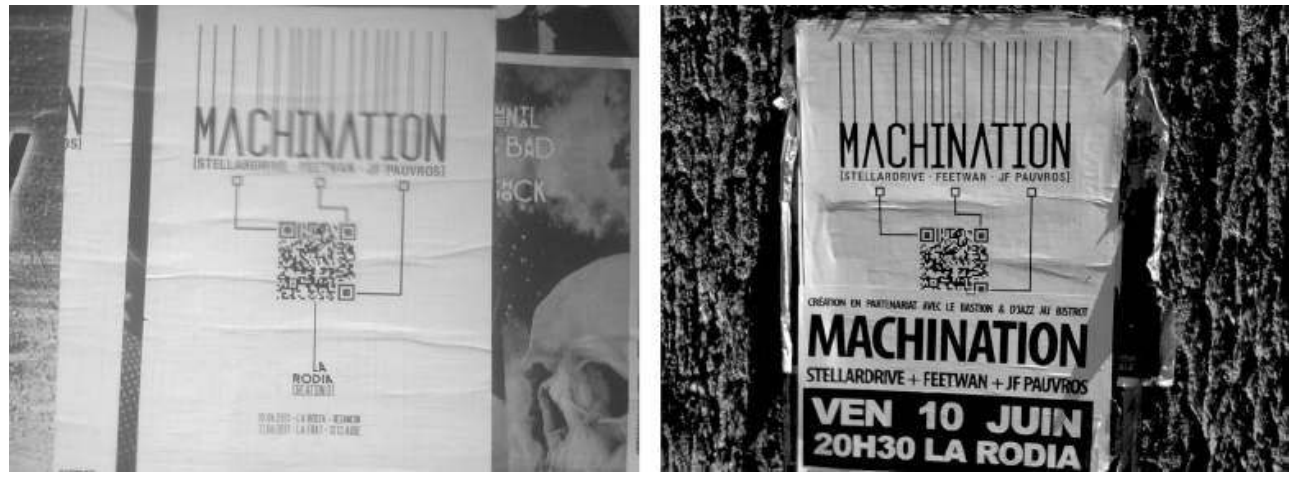

\section{QR-Code : des intentions de production aux effets sur la réception}

Le choix de Nicolas Chevailler pour le medium code-barres en 2D est triplement motivé : contrairement à son prédécesseur le simple code-barres, il offre la possibilité d'y intégrer trois informations (en même temps ou non, selon les générateurs) : une adresse web, un texte et un numéro de téléphone. Aussi, ce moyen de communication est encore nouveau en France et ne manque pas d'interpeller le public. Et enfin, ses qualités graphiques le rendent très intéressant, laissant une certaine liberté de manipulation; fonctionnant sur le principe du contraste, il est par exemple possible de transformer un carré noir en carré rouge, d'intégrer un texte sans que la lisibilité du code-barres 2D ne soit menacée. Par 
ailleurs, N. Chevailler nous informe que le choix du QR-code (et non du flashcode) a été motivé par un aspect économique: son édition se fait gratuitement par le biais d'un générateur en ligne, contrairement au flashcode qui nécessite l'achat d'une licence. Comme nous avons pu l'observer précédemment, $\mathrm{N}$. Chevailler tente de créer une attente chez le public avec une communication sérielle et évolutive. Il pense cette campagne de communication sur le principe du teasing, dévoilant petit à petit l'objet de son discours. Il remarque à juste titre que cette stratégie a fonctionné; en effet, de nombreuses personnes ont appelé la salle de concerts pour avoir des informations. Cependant, le nombre de téléchargements, de visite des sites induits par l'activation du QR-code n'ont pu être mesurés, tout comme l'impact de cette campagne sur la décision des spectateurs d'assister à la représentation. En somme, l'aspect mystérieux du code-barres 2D a sans doute joué un rôle de captation, il a pu attirer le regard du passant qui a ensuite décidé de se renseigner sur ce qu'est pour lui un signe. Ce type de fonctionnement est proche de celui induit par l'affichage illicite analysé par J. Fontanille (2004), ce dernier jouant sur une certaine connivence avec son destinataire. Ce phénomène peut être considéré positivement: le code-barres 2D, de par son originalité fait-faire, mais également négativement: ce medium ne remplit pas son rôle informatif. Et $\mathrm{N}$. Chevallier le reconnaît : ce type de communication est "élitiste», ou du moins segmentant. Elle ne s'adresse a priori ni aux enfants ni aux personnes âgées. Son public cible fait même partie de ce qu'on appelle les geeks, il doit maitriser une technologie relativement nouvelle, il a un savoir, un savoir-faire et un pouvoir-faire (fourni par les qualités du photophone) supérieurs dans ce domaine ${ }^{12}$. Pour N. Chevailler, cette campagne a également un rôle éducatif. L'insertion d'instructions au milieu du QR-code a une fonction de guide. Elles sont là pour inciter celui qui possède déjà la technologie à l'utiliser. N. Chevailler souligne également que ce type de communication toucherait un public plus large avec des budgets plus conséquents permettant la diffusion dans de plus gros formats. Il cite l'exemple du groupe britannique Mogwaï qui a basé la promotion de sa tournée uniquement sur des QR-codes. Enfin, le graphiste souligne que la réussite d'une telle campagne dépend de la capacité d'implication, de la curiosité du public et du respect par les concepteurs de certaines contraintes: les sites sur lesquels les codes-barres 2D renvoient ne doivent pas utiliser du flash par exemple, ce format n'étant pas pris en charge par les téléphones mobiles. Enfin, et c'est ce que nous avons pu constater en analysant rapidement la dernière affiche, l'utilisation des codes-barres 2D entre en résonance avec la thématique de l'évènement, crée une cohérence entre les valeurs du spectacle et celles de la campagne de publicité, allant jusqu'à lui donner une place de choix voire l'intégrer dans la création. Il est évident ici que l'utilisation des codesbarres 2D permet de dépasser une communication informationnelle par voie d'affichage, lui ajoutant une dimension ludico-esthétique non négligeable.

\section{Conclusion}

16 En France, les codes-barres 2D sont essentiellement utilisés pour informer et pour vendre, c'est-à-dire pour leur aspect simplificateur, pratique (donner accès en quelques clics à une information actualisée) et pour leur force de séduction, leur capacité à faire croire à leur utilisateur en l'obtention de valeurs euphoriques, telle une hausse de savoir rapide et exclusive. Le code-barres 2D est un medium puissant, il donne à ses utilisateurs le pouvoir d'ubiquité (virtuelle), et est donc de plus en plus utilisé parce qu'il intrigue de par sa 
nouveauté, parce qu'il offre une certaine liberté à son concepteur comme à son utilisateur. C'est un contenant mais ce n'est pas qu'un support car le message qu'il délivre ne se résume pas au contenu qu'il abrite, auquel il donne accès. Il rend l'utilisateur acteur, voire co-producteur. Ce phénomène n'est pas nouveau en soi, A.-M. Dujarier ( op. cit.) en fait état à travers une multiplicité de situations (du meuble en kit au logiciel à installer soi-même). Dans les cas envisagés ici, on ne peut identifier de bénéficie direct (entendre un gain d'argent) pour l'émetteur. Cependant, nous souhaitons souligner que cette technologie pousse de manière relativement masquée le simple utilisateur à devenir consommateur, à adhérer à un événement en achetant sa place en ligne par exemple et ce, en activant un simple lien.

17 En somme, le code-barres 2D ajoute ses propres valeurs au message, c'est-à-dire modernité, efficacité et originalité, le transformant. Et ceci est valable dans ses utilisations marketing comme dans ses utilisations détournées à des fins subversives, tel le travail de RaspouTeam ${ }^{13}$ dispersant des QR-codes dans la ville de Paris, rappelant ainsi aux promeneurs attentifs des événements historico-politiques marquants mais peu connus. Nous ne pouvons que constater un développement de plus en plus massif et de plus en plus divers dans l'utilisation des codes-barres 2D (publicité, légende dans les musées, temps d'arrivée du prochain métro...). Ils rappellent sur quelques points l'intégration dans des publicités murales du métro parisien du bluetooth, technologie qui envoie aux téléphones portables passant à proximité de l'affiche un message incitant à en savoir plus sur un produit, un service... Le code-barres 2D semble moins intrusif mais il relève cependant également « d'un régime de disponibilité visuelle qui invite à les traiter par défaut sur le mode de la vigilance périphérique ", le "choix de s'engager dans un traitement attentionnel plus approfondi semble laissé à l'initiative de l'usager ». (Licoppe, 2009, p. 38). Il serait donc sans doute désormais intéressant de confronter ces observations relatives à ce qui est mis en place par le dispositif avec une étude quantitative et qualitative de sa réception.

\section{BIBLIOGRAPHIE}

M. Amri, A.-L. Cano, M.-J. Catoir, E. Lechenaut et M. Versel, (2007), Un nouveau regard sur l'affichage urbain : le passage Saint Rémy à Bordeaux, http:// communicationorganisation.revues.org/367, date de la dernière visite : le 24 août 2011.

Beauchamps M., (2007), Internet : vers une fragmentation de la ville?, http:// communicationorganisation.revues.org/340, date de la dernière visite : le 24 août 2011.

Bourreau M. et Gensollen M., (2006), L'impact d'Internet et des Technologies de l'Information et de la Communication sur l'industrie de la musique enregistrée, http://rei.revues.org/459, date de la dernière visite : le 24 août 2011.

Chirollet J.-C., (2011), La question du détail et l'art fractal (à bâtons rompus avec Carlos Ginzburg), Paris, L'Harmattan, 280 p. 
Cloutier J., (1973), L'audiovisuel remis en question, in Communication et Langages $n^{\circ} 41-42$, pp. 39-51.

Courtès J., (1991), Analyse sémiotique du discours, de l'énoncé à l'énonciation, Paris, Éditions Hachette Supérieur, $302 \mathrm{p}$.

Dujarier M.-A., (2008), Le travail du consommateur, Paris, La Découverte, 246 p.

Eychenne F., (2009), La ville 2.0, complexe et familière, Limoges, Fyp éditions, 96 p.

Floch J. M., (1989), La contribution d'une sémiotique structurale à la conception d'un hypermarché, RAM, Recherche et Application en Marketing, vol. 4, n², pp. 37-49.

Fontanille J., (2004), Affichage : de la sémiotique des objets à la sémiotique des situations, http:// www.ec-aiss.it/index_d.php?recordID=121, date de la dernière visite : le 25 août 2011.

Jauréguiberry F. et Proulx S., (2011), Usages et enjeux des technologies de communication, Toulouse, éditions érès, $144 \mathrm{p}$.

Licoppe C. et Levallois-Barth C., (2009), Configurer l'accessibilité des voyageurs équipés à des services mobiles multimédia : le cas des publicités bluetooth dans le métro parisien, in Réseaux volume 27-156, pp. 15-48.

Licoppe C. et Zouinar M., (2009), Les usages avancés du téléphone mobile, in Réseaux volume 27-156, Paris, La Découverte, 285 p.

Mitropoulou E., (2008), Vers une sémiotique du medium : une problématique à légitimer ?, http:// revues.unilim.fr/nas/document.php?id=2730, date de la dernière visite : le 26 juin 2012.

\section{NOTES}

1. Les chiffres avancés ici sont à relativiser, créée en juin 2005 par Bouygues Telecom, Orange France, SFR, le GESTE et l'ACSEL, l'Association Française du Multimédia Mobile regroupe les principaux acteurs du marché du multimédia mobile en France. Il nous parait donc souhaitable pour cette association de mettre en évidence voire d'exagérer l'ampleur du phénomène.

2. Mobile Content Forum se donne pour mission d'informer les entreprises implantées en dehors $\mathrm{du}$ Japon des derniers développements [nippons] en matière de mobile.

3. Gratuitement, sur le site http://www.y01.fr/ par exemple.

4. Ainsi, un homme a choisi de se faire tatouer un «EZ code», type de code-barres 2D donnant accès à une vidéo complétant et animant son tatouage (http://www.youtube.com/watch?

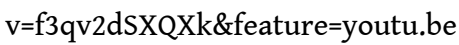

5. Le medium est d'un niveau axiologique supérieur au support, qu'il englobe, il veut communiquer ou «manipuler » (dans le sens de Courtès) : «Le medium mass-media se présente alors comme ce qui fait-être un faire-faire et un faire-croire» (Mitroupoulou, 2008).

6. Dans cet article, E. Mitropoulou cherche à poser les bases d'une « sémiotique du medium ». Elle qualifie le medium de nouveau pour signifier qu'il n'était pas considéré comme tel jusque là (soit parce qu'on parlait de support, soit par qu'il n'existait pas...).

7. Les codes-barres 2D n'ont en effet pas forcément besoin de connexion Internet WIFI pour être activés, ils utilisent les réseaux mobiles comme EDGE ou la 3G.

8. Cette création est le fruit de la rencontre entre Stellardrive (post-rock), Fetwan (drum'n bass) et J.-F. Pauvros (free jazz). Après une semaine de résidence à la Rodia, SMAC de la ville de Besançon, la création est présentée au public bisontin le 10 et à St Claude (39) le 11 Juin 2011.

9. Nicolas Chevailler est également membre du groupe Stellardrive et intervenant en communication dans une école supérieure de commerce bisontine. 
10. N. Chevailler précise que selon lui, il vaut mieux n'utiliser qu'un $Q R$-code par affiche, donnant accès à toutes les informations possibles, plutôt que deux apportant chacun une information, car l'affiche s'en trouvera moins visible.

11. http://www.stellardrive-music.org/machination/.

12. Ainsi, les remarques émises par M. Beauchamp (2007) au sujet de la fragmentation de la population au regard de son usage d'Internet sont d'autant plus valables avec les codesbarres 2D, ces derniers appartenant aux Nouvelles Technologies de l'Information et de la Communication.

13. http://www.raspouteam.org/QR/.

\section{RÉSUMÉS}

Petit carré composé d'une suite d'espaces noirs et blancs, le code-barres 2D permet, avec l'aide d'un téléphone mobile adéquat, d'avoir accès à une quantité d'informations. Encore peu utilisé en France, il est cependant de plus en plus présent dans notre quotidien. Cet article propose, à travers l'étude de deux cas particuliers de marketing, d'évaluer le statut et les valeurs associées à cet objet en fonction des pratiques qu'il est censé induire.

Two-dimensional barcodes made of black modules arranged in a square pattern on a white background allow access to a great deal of information using a suitable mobile phone. Although these are not widely used in France, they are becoming increasingly present in daily life. Through the study of two marketing cases, this paper proposes to assess the status and values associated with this technology depending on its potential uses.

\section{INDEX}

Mots-clés : medium, code-barres 2D, NTIC, sémiotique, affichage, campagne de communication, marketing

Keywords : medium, two-dimentional code, ICT, semiotic, advertising campaign, marketing

\section{AUTEUR}

\section{VÉRONIQUE MADELON}

CIMEOS -

Université de Bourgogne 